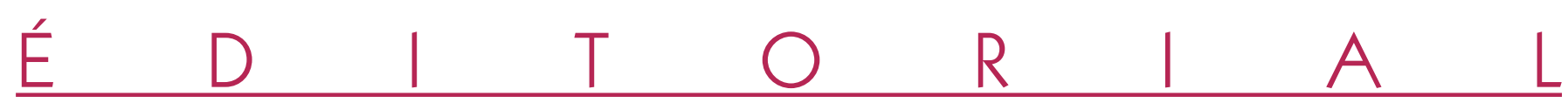

\title{
A propos du développement professionnel continu
}

e développement professionnel continu est une réalité depuis le 1er janvier 2013. De nombreuses prises Lde position se sont exprimées en défaveur de cette nouvelle approche de la formation continue.

Certes un travail de grande qualité a été effectué dans le domaine de la formation avec le CNFCO, qui a incité nos confrères et consoeurs à prendre conscience de l'obligation d'apprendre, de comprendre et de modifier leur exercice quotidien en fonction de l'évolution des connaissances et de la mutation de notre société.

Le DPC arrive ! Ce nouveau dispositif réglementaire n'est pas une révolution mais une évolution.

Pratiquement le DPC a pour objectif l'analyse des pratiques professionnelles, le perfectionnement des connaissances, l'amélioration de la qualité et de la sécurité des soins et la prise en compte des priorités de santé publique.

Le DPC n'est qu'un aspect obligatoire de la formation continue, mais ne se substitue pas à la formation, délivrée dans nos congrès ou organisée par nos sociétés scientifiques sur le terrain avec une grande qualité.

Des expériences de DPC ont été mises en ouvre dans le cadre du congrès de l'ADF depuis 2009. En Vendée, en Bretagne, des groupes d'évaluation des pratiques professionnelles (EPP) fonctionnent depuis plusieurs années. Le retour des impressions des confrères a toujours été positif.

Les 5 grandes étapes d'un programme DPC sont:

- l'analyse des pratiques (obligatoire avant la journée avec présence effective). Le praticien remplit un questionnaire confidentiel de pré évaluation pour identifier ses besoins de formation et ouvre un plan d'action.

- L'acquisition des connaissances se fait lors d'une journée de formation en fonction des besoins du groupe. 
- La mise en œuvre du plan d'action permet au praticien de retour au cabinet d'adapter sa pratique aux recommandations proposées.

- L'analyse des pratiques (2 à 3 mois après la journée de présence effective) : le praticien répond à un nouveau questionnaire pour évaluer la progression et les points éventuels d'optimisation.

- La validation du DPC (sur www.mondpc.fr) déclenchera l'attestation de fin de parcours et l'indemnisation du praticien.

Certes ce dispositif va évoluer au fur et à mesure des expériences. Aujourd'hui il apparait un peu lourd, beaucoup de questions concernant le dispositif administratif demeurent sans réponses, en particulier celles concernant son financement.

Nous devons poursuivre notre effort de formation au-delà de cette simple obligation pour obtenir une reconnaissance nécessaire à notre équilibre psychique quand l'actuelle logique de performance ne nous signifie que notre insuffisance, ou nourrir notre motivation en proposant sans cesse de nouveaux défis, ou évacuer la confrontation à l'autre en remplaçant le conflit par la compétition.

Le DPC s'impose, apprenons à en faire bon usage!

\author{
Christian Decloquement \\ Président du collège de Bonnes Pratiques \\ en médecine bucco-dentaire \\ Secrétaire général adjoint de l'ADF
}

Les opinions émises n'engagent que leurs auteurs. 